\title{
Coexisting Pituitary Adenoma and Suprasellar Meningioma: A Coincidence or Causation Effect? Report of Two Cases and Review of Literature
}

\author{
Seyed-Abolghasem Mortazavi ${ }^{1}$, Mohamad Shirani ${ }^{1}$, Saeed Saeedinia ${ }^{1}$, Reza Sanjari ${ }^{1}$, Hamed Hanif ${ }^{1}$, Abbas \\ Amirjamshidi ${ }^{*}$ \\ ${ }^{1}$ Department of Neurosurgery, Sina Hospital, Tehran University of Medical Sciences (TUMS), Tehran, Iran \\ * Corresponding Author: MD, MPH, Professor of Neurosurgery, Department of Neurosurgery, Sina Hospital, Tehran \\ University of Medical Sciences (TUMS).Tel; 00982177523065, Fax; 00982177500958, E-mail:abamirjamshidi@yahoo.com
}

Received: November 7, 2014, Revised: January 24, 2015, Accepted: February 26, 2015

\section{Abstract}

Background and Importance: The coexistence of pituitary adenoma and another type of brain tumor is a very rare clinical scenario. Even though such a presentation can be an incidental event but the possible pathogenesis of coexistence of different lesions in the sella and suprasellar region has not yet been elucidated.

Case Presentation: Two cases of concomitant sellar and suprasellar region tumors are reported. A 37-year-old lady with Prolactinoma and a suprasellar meningioma and a 42-year-old Acromegalic man with suprasellar meningioma and a pituitary adenoma (PA).

Conclusion Both meningiomas were removed transcranially while the Prolactinoma could be managed medically and the growth hormone $(\mathrm{GH})$ secreting adenoma was removed trans-sphenoidally. The visual problems and hormonal imbalances of both patients improved postoperatively and there has been no sign of recurrence of the lesions after a mean of five years follow up. The literature is reviewed on this topic and the possible pathogenesis and management protocol of similar lesions are discussed.

Keywords: Acromegaly; Multiple primary brain tumor; Prolactinoma; Suprasellar meningioma; Pituitary adenoma

Please cite this paper as: Mortazavi S.A, Shirani M, Saeedinia S, Sanjari R, Hanif H, Amirjamshidi A. Coexisting Pituitary Adenoma and .Suprasellar Meningioma: A Coincidence or Causation Effect? Report of Two Cases and Review of Literature. Iran J Neurosurg. 2015;1(1):43-46

\section{Background \& Importance}

Concomitant brain tumor and association of PA with other tumors comprise $15-25 \%$ of all intracranial neoplasms with an annual incidence of 6/100,000 (1). Pituitary adenomas (PAs) are very common benign neoplasms, with a prevalence of 10 to $23 \%$ in unselected adult population considering population surveys, radiological, and autopsy series $(2,3)$. The coexistence of PA and another type of brain tumor is a very rare clinical scenario $(4,5)$. The type of PAs reported in such series varied from, clinically silent lesions "non-functional" to functioning adenomas such as prolactinomas and growth hormone (GH)and thyrotropin (TSH)-secreting tumors (1,4,6-9).

In cases of PA concurrent with meningioma, GH-secreting adenoma is the most predominant (9-11). Even though prolactinomas represent the most common type of PA in adults (up to 70\%), the association of this type of PA with two or more primary brain tumors is a relatively rare occurrence.

The pathogenesis of coexistence of different lesions in the sella and suprasellar region has not been elucidated. Concurrent adjacent double tumors in other locations of central nervous system (CNS) are even more implying the effect of unknown oncogenic factors (7).

In this communication, we do not intend to report collision 'intrasellar' pathologies (12-14) but two cases of concomitant brain tumors (CCBT) are reported. Both tumors occurred in the sellar region. The adenomas were one prolactinoma and one $\mathrm{GH}$-secreting tumor. The literature is reviewed on this topic and even though this situation can be a co-incidence, we will discuss the possible pathogenesis and management protocol of similar lesions.

\section{Case Presentation}

\section{Case 1}

A 37-year-old lady presented with 8 month history of oligomenorrhea and spotting. She was referred to a gynecologist and received LD tablet (Ovocept-LD, Aburaihan Co.). Headache, diplopia, progressive visual impairment and persistent oligomenorrhea were the main causes of referring her to the neurosurgery department.

In physical examination, left partial third nerve paresis and 
decreased visual acuity (VA) to 80. 200 in Sentinel scale with a left temporal hemianopia were the only remarkable findings. The non-contrast enhancing computed tomography (CT) scan performed in the other department showed iso- to hypo- dense supra sellar lesion. Magnetic resonance imaging (MRI) revealed a well-delineated round tumor $30 \times 25 \times 20 \mathrm{~mm}$ in diameter, T1W isointense and $\mathrm{T} 2 \mathrm{~W}$ hyperintense lesion located within the sella turcica and another dural based lesion lying over the planum sphenoidale and diaphragm sella. The intrasellar lesion showed a faint enhancement after contrast material injection but the suprasellar lesion had a bright enhancement with the base of the lesion extending over the jugum sphenoidale and dighragm sella in the meantime (Figure 1). Pituitary function tests were all normal except for a serum prolactin level of $840 \mathrm{ng} / \mathrm{dl}$ (3$31 \mathrm{ng} / \mathrm{dl})$. She was conservatively managed with Cabergoline (Dostinex tablet $0.5 \mathrm{mg}$, Pfizer Co.) during the previous 4 months without any improvement in her vision. It was decided to decompress the optic apparatus through a right pterional craniotomy and total removal of the suprasellar tumor could be performed using microsurgical technique. Diaphragm sella was intact and we did not attempt to excise the intrasellar lesion. Improvement of her vision was remarkable in the postoperative period and further medical treatment was undertaken using 1.5 mg Dostinex weekly.

\section{Case 2}

A 42-year-old man was referred to our department with the diagnosis of Acromegaly. Serum GH level was 65 IU (up to $5 \mathrm{IU}$ ) and IGF1 $890 \mathrm{IU}$ (up to $370 \mathrm{IU}$ ). His VA was decreased during the previous 6 months to 40.200 in Sentinel scale with bitemporal hemianopia. Imaging revealed an intrasellar lesion isointense in T1W and T2W images which was enhancing homogenously and mildly after contrast material injection. There was also a suprasellar lesion $3 \times 3 \times 2 \mathrm{~cm}$ in diameter with the same intensity I T1W and T2W images which enhanced notably after contrast material injection (Figure 2). It could not be

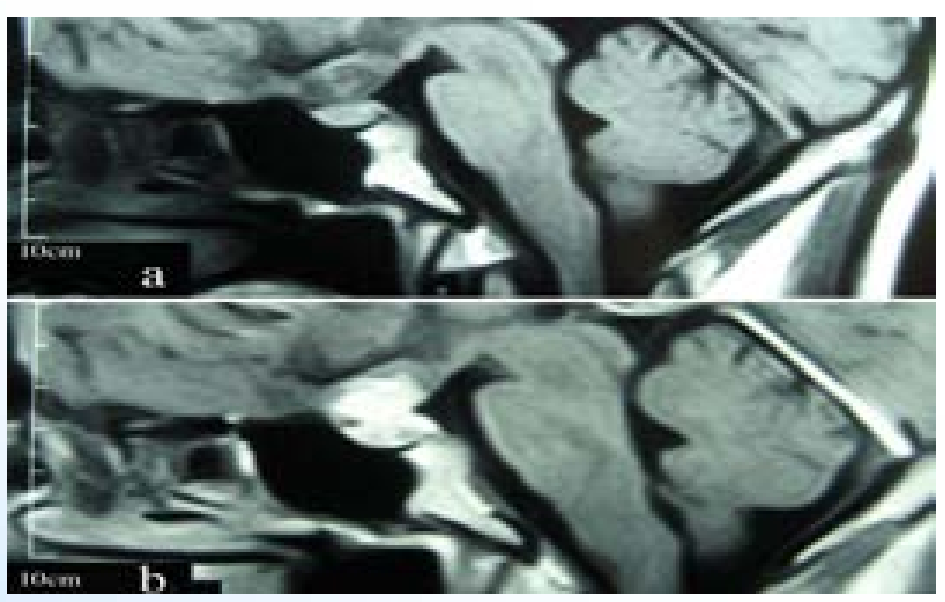

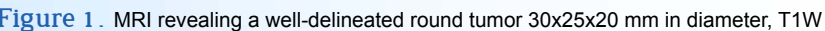
isointense and T2W hyperintense lesion located within the sella turcica and another dural based lesion lying over the planum sphenoidale and diaphragm sella. The intrasellar lesion showing a faint enhancement after contrast material injection but the suprasellar lesion has a bright enhancemen verified whether the lesions were of the same nature or 'double coexisting lesions'. Considering the acromegalic nature of the patient a transeptal transsphenoidal (TSSA) microscopic adenomectomy was performed and a pinkish gray soft PA could be easily removed from within the sella. The normal looking pituitary gland and intact diaphragm prohibited us from extending our search beyond the diaphragm sella. The overnight serum GH level was 10 IU. Considering no improvement in his VA, a right pterional craniotomy was performed and a purplish, lobulated, meaty tumor uplifting the chiasm and optic nerves could be excised gross totally. The diaphragm sella and jugum sphenoidale were the areas coagulated and curetted to achieve Simpson I tumor excision. The postoperative course was uneventful and VA improved remarkably. The patient has been under control in OPD during the previous $>9 \mathrm{y}$ without recurrence of any of the tumors either clinically or in the images.

\section{Discussion}

Coexisting PA and suprasellar meningioma is a rare occurrence. Our search in PubMed and Google Scholar and handhold identification of the references of each article revealed twelve similar cases reported in the available literature. The keywords used for this search were; 'pituitary adenoma ', 'supra sellar meningioma', 'multiple primary brain tumor' 'tuberculum sellae', 'collision tumors', 'CNS' and 'coexisting tumors'.

Definition and epidemiology, considering MESH terminology and related references in the literature, there have been different descriptions used to define coexistence of more than one tumor; 'collision tumors' are those with infiltration of a tumor by another type of tumor while 'coincidental tumors are synchronous tumors of different histogenesis in contiguous or far from each other (15). We would like to suggest that the coexisting tumors in cases like ours are most probably of coincidental type rather collision tumors.

Patients with neurocutaneous disorders such as Neurofibromatosis type I and II (NF1\&2) are predisposed to the develop meningiomas, various types of gliomas, schwannomas and neurofibromas (16). It is suggested that considering meningioma and pituitary adenoma are both common neoplasms of CNS, their occurrence in a single patient can be coincidental $(17,18)$.

Hypothetical Pathogenesis- Several mechanisms have been suggested for triggering development of multiple primary brain tumors of different histology in a single patient. Jan Fortuniak et al. believe these cases occur incidentally, and the etiology of this phenomenon remains unknown (19). K Suzuki et al. in their Immunohistochemical analysis showed that concurrent adjacent double tumors occur because of activation of the signaling pathways of receptor tyrosine kinases (20). According to Tugcu B et al. and Black et al. (2006) one tumor may secrete a growth factor that initiates growth of another lesion $(21,22)$. It is yet to be investigated whether in GH-producing adenoma, both the GH itself or the treating substance, such as Somatostatin, 


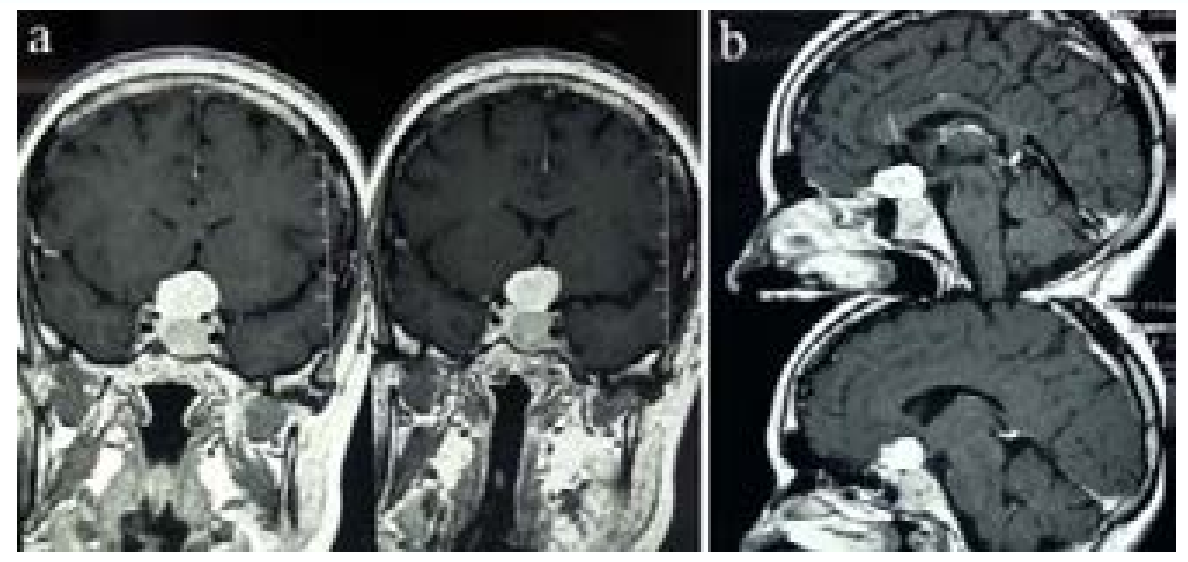

Figure 2. MRI showing a) an intrasellar lesion isointense in T1W and T2W images enhancing homogenously and mildly after contrast material injection and b) a suprasellar lesion $3 \times 3 \times 2 \mathrm{~cm}$ in diameter with the same intensity in T1W and T2W images which enhanced notably after contrast material injection.

might induce arachnoid cap cell transformation to meningioma (23). The other mechanisms hypothesized as causative for the development of multiple tumors in the CNS are exposure to the offending biochemical substances, genetic factors, prior trauma and surgery (16-18). Any compressive or destructive lesion involving the pituitary stalk can cause moderate hyperprolactinemia (up to150 ng/mL). This phenomenon frequently referred to as the stalk effect. As a consensus, a prolactin level in excess of $200 \mathrm{ng} / \mathrm{mL}$ might be the result of a PRL-producing tumor. In our case the serum prolactin level was $840 \mathrm{ng} / \mathrm{dl}$ and the stalk effect was almost excluded.

Treatment algorithms- It is important to distinguish between an adenoma with suprasellar extension and an adenoma coexisting with a suprasellar meningioma because the treatment strategy for these tumors is different and in different ways. Although no pathognomonic radiological characteristic is seen in imaging and no specific modality of MRI can differentiate definitely between these tumors, and some characteristic of suprasellar meningiomas such as bright homogeneous enhancement, intracranial dural base might distinguish them from pituitary adenomas $(24,25)$. Most of the authors suggest that both tumors should be removed in one session (26). If removal of the tumors is not possible in one session, it is important to decide which tumor should be operated on first (22). YJ Lu et al. (2007) suggested that avoiding the complications associated with the transcranial approaches, extended transsphenoidal surgery might be a good alternative choice for approaching suprasellar meningioma and pituitary adenoma simultaneously (20). The ideal approach for treatment of such concomitant tumors is not clear due to lack of adequate experience in the literature $(5,11,13,22-24,27-32)$. Visual impairment was the main remaining complaint of both our patients after attempting to treat the PA either medically or surgically. We proceeded to decompress the suprasellar component of the tumor transcranially in both cases which turned to be suprasellar meningioma.

\section{Conclusion}

In our experience, it is suggested; a) using high quality imaging with good resolution and specified techniques can preclude loosing golden time for preservation of vision in these CCBT, b) surgical approach should be tailored according to the individual patient's symptom, the anatomical characteristics of the mid skull base region and the feasibility of resection all two tumor in one session for the attending surgeons.

\section{Funding}

None declared

\section{Conflicts of Interest}

The authors have no conflict of interest.

\section{References}

1. Furtado SV, Venkatesh PK, Ghosal N, Hegde AS. Coexisting intracranial tumors with pituitary adenomas: Genetic association or coincidence? Journal of cancer research and therapeutics. 2010;6(2):221.

2. Daly AF, Tichomirowa MA, Beckers A. The epidemiology and genetics of pituitary adenomas. Best Practice \& Research Clinical Endocrinology \& Metabolism. 2009;23(5):543-54.

3. Guaraldi F, Prencipe N, di Giacomo V, Scanarini M, Gasco V, Gardiman MP, et al. Association of craniopharyngioma and pituitary adenoma. Endocrine. 2013;44(1):59-65.

4. Mathuriya S, Vasishta R, Dash R, Kak V. Pituitary adenoma and parasagittal meningioma: an unusual association. Neurology India. 2000;48(1):72.

5. O'Connell JE. Intracranial meningiomata associated with other tumours involving the central nervous system. British Journal of Surgery. 1961;48(210):37383

6. Curto L, Squadrito S, Almoto B, Longo M, Granata F, Salpietro F, et al. MRI finding of simultaneous coexistence of growth hormone-secreting pituitary adenoma with intracranial meningioma and carotid artery aneurysms: report of a case. Pituitary. 2007;10(3):299-305.

7. da Costa Jr LB, Riva-Cambrin J, Tandon A, Tymianski M. Pituitary adenoma associated with intraventricular meningioma: case report. Skull Base. 2007;17(5):347.

8. Fernandez A, Karavitaki N, Ansorge O, Fazal-Sanderson V, Wass JA. Acromegaly and anaplastic astrocytoma: coincidence or pathophysiological relation? Pituitary. 2008;11(3):325-30

9. Laun A, Lenzen J, Hildebrandt G, Schachenmayr W. [Tuberculum sellae meningioma and hypophyseal adenoma in a woman]. Zentralblatt fur Neurochirurgie. 1992;54(3):119-24.

10. Brennan Jr TG, Rao CK, Robinson W, Itani A. Tandem Lesions: Chromophobe Adenoma and Meningioma. Journal of computer assisted tomography. $1977 ; 1(4): 517-20$

11. Bunick EM, Mills LC, Rose LI. Association of acromegaly and meningiomas. JAMA. 1978;240(12):1267-8. 
12. Amirjamshidi A, Amiri RS, Alimohamadi $M$, Abbassioun K. Concomitant intraventricular colloid cyst and low-grade astrocytoma of the brainstem in a 16-year-old boy: Case report. Journal of Neurosurgery: Pediatrics. 2011;8(4):342-5

13. Khalatbari $M$, Borghei-Razavi $H$, Shayanfar $\mathrm{N}$, Behzadi AH, Sepehrnia A. Collision tumor of meningioma and malignant astrocytoma. Pediatric neurosurgery. 2009;46(5):357-61.

14. Koutourousiou M, Kontogeorgos G, Wesseling P, Grotenhuis AJ, Seretis A. Collision sellar lesions: experience with eight cases and review of the literature. Pituitary. 2010;13(1):8-17.

15. Buccoliero AM, Taddei GL, Caldarella A, Mennonna $\mathrm{P}$, Ammannati $\mathrm{F}$, Taddei $\mathrm{A}$, et al. Meningioma-primary brain lymphoma association. Neuropathology. 2004;24(4):336-40

16. Prayson RA Chowdhary $S$, Woodhouse S, Hanson M, Nair S. Collision of a syncytial meningioma and malignant astrocytoma. Annals of diagnostic pathology. 2002;6(1):44-8.

17. Matyja E, Kuchna I, Kroh H, Mazurowski W, Zabek M. Meningiomas and Gliomas in Juxtaposition: Casual or Causal Coexistence?: Report of Two Cases. The American journal of surgical pathology. 1995;19(1):37-41.

18. Probst A. Kombination eines CushingSyndroms, Hypophysenadenoms und suprasellären Meningeoms-Fallbericht. Z Zbl Neurochir. 1971;32:75.

19. Fortuniak J, Jaskólski DJ, Zawirski M, Tybor K. Glioblastoma multiforme and meningioma in the same patient-a case report. Neurologia neurochirurgia polska. 2006;41(3):272-5

20. Suzuki K, Momota H, Tonooka A, Noguchi H, Yamamoto K, Wanibuchi M, et al. Glioblastoma simultaneously present with adjacent meningioma: case report and review of the literature. Journal of neuro-oncology. 2010;99(1):147-53.

21. Black PM, Carroll R, Glowacka D, Riley K, Dashner K. Platelet-derived growth factor expression and stimulation in human meningiomas. Journal of neurosurgery. 1994;81(3):388-93.

22. Tugcu B, Kepoglu U, Gunal M, Gunaldi O, Karakaya B, Demirgil BT. Two distinct primary brain tumors, in same region of the same patient: a case report. Journal of neuro-oncology. 2006;79(2):219 20

23. Abs $R$, Parizel $P$, Willems $P$, Van de Kelft E, Verlooy J, Mahler $\mathrm{C}$, et al. The association of meningioma and pituitary adenoma: report of seven cases and review of the literature. European neurology. 1993;33(6):416-22.

24. Lu Y-J, Chuang C-C, Jung S-M, Wei K-C. Case Report Synchronous Pituitary Adenoma and Tuberculum Sellae Meningioma. Neurosurgery. 2007;60(4 Suppl 2):E401; discussion E401.

25. Wild Kv, von Ruf $\mathrm{H}$. Diagnostic problems and errors in suprasellar meningiomas. Modern Aspects of Neurosurgery Excerpta Medica Amsterdam. 1974:43.

26. Spallone A, Santoro A, Palatinsky E, Giunta F. Intracranial meningiomas associated with glial tumours: a review based on 54 selected literature cases from the literature and 3 additional personal cases. Acta neurochirurgica. 1991;110(3-4):133-9.

27. Görge H, Pöll W, Gers B. [Para-and suprasellar meningioma coincident with a hormonally active intrasellar hypophyseal adenoma--case report] Zentralblatt fur Neurochirurgie. 1992;54(4):190-6.

28. Hainer V, Krejcik L, Pelikan J, Tvaroh F, Urbanek J. Meningioma in contact with eosinophilic adenoma in a patient with acromegaly (author's transl). Casopís lékařů českých. 1978;117(27):829.

29. Jaskolski D, Jakubowski J. Association of suprasellar meningioma with pituitary adenoma. Zentralblatt fur Neurochirurgie. 1989;51(4):229-31.

30. Poeata I, Besson G, lliescu B, Faiyad Z, Indrei A Dumitrescu G-F. Meningioma association with three different cell types tumors: report of clinical cases and review of the literature. Romanian Neurosurgery. 2010;1:46-51.

31. Prevedello DM, Thomas A, Gardner P, Snyderman $\mathrm{CH}$, Carrau RL, Kassam AB. Endoscopic endonasa resection of a synchronous pituitary adenoma and a tuberculum sellae meningioma: technical case report. Neurosurgery. 2007;60(4):E401.

32. Zentner J, Gilsbach J. Pituitary adenoma and meningioma in the same patient. European archives of psychiatry and neurological sciences $1989 ; 238(3): 144-8$

\section{Comments}

Mortazavi and colleagues are reporting two coinciding pituitary adenomas and supra stellar meningiomas (1). They have successfully managed the meningiomas by craniotomies. The concomitant pituitary lesions were managed trans-sphenoid ally in the acromegalic case and medically in the prolactinoma case. This coincidence although known since very old, however it is a rarity to deserve the report. We still need more knowledge about the clinical course in long run of such rumors. We shall look forward to see the follow up repots of the ceases.

Hooshang Saberi, MD, MPH, Associate Professor of Neurosurgery, Tehran University of Medical Sciences, Tehran, Iran

Reference:

1.Mortazavi S.A, Shirani M, Saeedinia S, Sanjari R, Hanif $\mathrm{H}$, Amirjamshidi A Coexisting Pituitary Adenoma and Suprasellar Meningioma: A Coincidence or Causation Effect? Report of Two Cas A Coincidence and Review of Literature. IJJNS. 2015;1(1):43-46 